\title{
A Retrospective Study of Malaria Trend in Libokemkem District Over the Last Five Years: North West Ethiopia
}

\author{
Lemma Workineh (iD) \\ Setegn Mekuria ${ }^{2}$ \\ Teklehaimanot Kiros (D) \\ Wasihun Hailemichael (ID) \\ Tahir Eyayu (1) ${ }^{\prime}$ \\ 'Department of Medical Laboratory, \\ Debre Tabor University, Debre Tabor \\ Town, Ethiopia; ${ }^{2}$ Department of Medical \\ Laboratory, Addis Zemen Health Center, \\ Addis Zemen Town, Ethiopia
}

Correspondence: Lemma Workineh Email lemmiwork@gmail.com
Background: Malaria is a vector-borne disease caused by public health important Plasmodium species. Despite the fact that Ethiopia has implemented several malaria prevention and control techniques aimed at reducing its morbidity and death, it continues as major cause of morbidity and mortality in Ethiopia. Transmission dynamics are really critical for guiding the selection of the appropriate intervention in a given area. As a result, the goal of this study was to analyze the trend of malaria prevalence over the last five years in the Addis Zemen health center.

Methods: An institutional-based retrospective analysis on malaria data from 2015/16 to 2019/ 20 was undertaken in Addis Zemen health center of Libokemkem district. The data collectors thoroughly and systematically collected the results of 15,452 blood films performed over a fiveyear period from the malaria registration book in the health center. Any data missing species and stage of the parasite, as well as sociodemographic characteristics, date, month, and year of blood film performed, were omitted from the study. Finally, data were entered and analyzed using SPSS version 25, with P-values of less than or equal to 0.05 deemed statistically significant variables. Results: The overall malaria slide positivity rate over the last five years in the study area was $10.9 \%$. From $2015 / 16$ to $2018 / 19$, the positive rate fell, then it spiked in 2019/20. Plasmodium falciparum was the most common parasite found, accounting for $72.6 \%$ of the total. The months of October, November, May, June, August, and September showed the largest number of malaria cases. Males, rural residents, and adult population groups were more affected by malaria, according to multivariate logistic regression $(\mathrm{P} \leq 0.05)$.

Conclusion: There was high malaria morbidity case in $2019 / 20$ as compared to the remaining years. Therefore, malaria control, prevention, and intervention programs in the district should be strengthened.

Keywords: malaria trend analysis, malaria slide positivity rate, Libokemkem district

\section{Background}

Malaria is a serious vector-borne disease caused by Plasmodium species (Plasmodium spp) that affects millions of people worldwide. ${ }^{1}$ It is most common in areas with a tropical temperature and plenty of stagnant water, which is ideal for mosquito breeding. ${ }^{2}$ Plasmodium falciparum (P. falciparum), Plasmodium vivax (P. vivax), Plasmodium malaria ( $P$. malaria), Plasmodium ovale( $P$. ovale), and the newly identified zoonotic parasite Plasmodium Knowlesi (P. knowlesi) are the five medically significant Plasmodium spp that cause human malaria. ${ }^{3}$ P. falciparum is the deadliest and most virulent Plasmodium spp, accounting for $50 \%$ of all malaria cases worldwide and $75 \%$ in Sub-Saharan Africa. ${ }^{4}$ 
The WHO world malaria reports, estimated cases and deaths for the 2020 years, were 229 million and 409, 000, respectively. ${ }^{5}$ The African continent accounts for $94 \%$ of all malaria cases worldwide. ${ }^{5}$ Malaria remains Ethiopia's most serious public health problem resulting in high levels of mortality and morbidity. ${ }^{6}$ About 3/4 of Ethiopia's land mass and $2 / 3$ of the country's population is projected to be at risk of malaria due to climatic and ecological conditions favorable for its transmission. ${ }^{6}$

It has been the major cause of morbidity and mortality for many years and affects four to five million people annually in the country. ${ }^{7}$ The disease causes 70,000 deaths each year and accounted for $17 \%$ and $15 \%$ of outpatient and inpatient visits to health institutions. ${ }^{8}$ Malaria is ranked as the leading communicable disease in Ethiopia, accounting for about $30 \%$ of the overall disability adjusted life years lost and making it a significant impediment to social and economic development. ${ }^{9}$

According to a report from the Ethiopian Federal Ministry of Health, out of 1,620,885 suspected cases, $25 \% \quad(410,409)$ tested positive for malaria, with P. falciparum accounting for $65 \%$ of positive cases and $P$. vivax accounting for the remainder over the previous five years. ${ }^{10}$ Since malaria transmission is seasonal and variable in Ethiopia, protective immunity is often inadequate, and people of all ages are at risk of contracting the disease. ${ }^{11}$

Despite the fact that Ethiopia has implemented several malaria prevention and control methods with the goal of reducing malaria morbidity and death, malaria remains a serious public health problem in the country as a whole and in the study area specifically. The main obstacles to controlling and preventing malaria are epidemicity owing to seasonal transmission, poor health care system like low coverage of insecticide treated nets (ITNs), drug resistance malaria due to selftreatment or incorrect species identification by microscopist, asymptomatic malaria cases, and Anopheles mosquito resistance to pesticides and chemicals. ${ }^{12,13}$

Analyzing the malaria morbidity pattern in a malariaaffected area would aid in understanding the dynamics of disease transmission and evaluating the efficacy of malaria intervention programs in reducing disease burden in a given area. Malaria is one of the most important public health issues in the Libokemkem district, particularly in the Addis Zemen catchment areas. According to information collected from health workers working at the Addis Zemen health center, it was detected in nearly half of the febrile patients. In the health center, no other study has been conducted on the trend of malaria so far. As a result, studying the five-year malaria trend in the area is important for providing information on malaria control interventions and evaluating the performance of the malaria elimination program. As a result, the study's goal was to close the gap on the patterns of malaria prevalence for better control and intervention in the disease.

\section{Materials and Methods Study Area}

A study was undertaken in the Libokemkem district's Addis Zemen health center. Addis Zemen is located between Bahir Dar and Gonder in the Amhara regional state, South Gonder Zone. It is the administrative center of the Libokemkem district and 637 kilometers from Ethiopia's capital city. The district sits at a height of 2000 feet above sea level. It is located between the latitudes of $11057^{\prime} 44.6^{\prime \prime} \mathrm{N}$ and $12025^{\prime}$ $32.6{ }^{\prime \prime} \mathrm{N}$, and the longitudes of $37034^{\prime} 4.89^{\prime \prime} \mathrm{E}$ and $3803^{\prime}$ 30.9'E. West Belessa Woreda in the North, Ebinat Woreda in the East, Fogera district in the South, and Lake Tana and Gonder Zurie district in the West bound the district according to the LiboKekem District Agriculture and Rural Development Office. The total area of the district is $1081.57 \mathrm{~km}^{2}$ sub-divided by 34 kebeles, of which 29 are rural kebeles and the rest are urban kebeles including the capital Addis-Zemen town (Figure 1).

According to Ethiopia's 2007 population and housing census, ${ }^{14}$ the Libo-kemkem district has a total population of 198,374, with 100,951 males and 97,423 females. Woinadega, Dega, and Qolla are the three main agroclimatic zones in the district, accounting for $73 \%, 22 \%$, and $5 \%$ of the total area, respectively. The district's typical annual rainfall ranges from 900 to $1400 \mathrm{~mm}$, with temperatures ranging from $18^{\circ} \mathrm{C}$ to $25^{\circ} \mathrm{C}$. In the district, both $P$. falciparum and $P$. vivax are the only malaria parasites reported. Five anopheline species including Anopheles gambiae, Anopheles cinereus, Anopheles demeilloni, Anopheles christi and Anopheles pretoriensis were reported as vector of malaria in the study area. ${ }^{15}$

The major transmission season of malaria follows the heavy rainfall (August, September, October and November) and the minor transmission season starts after minor shower (April to May) in the area. Treating of the parasite with anti malarial chemotherapy and ITN is the prevention and control strategy used in the district. Due to its low land type and climatic element, Addis Zemen is one of the malaria-prone areas in the district. 

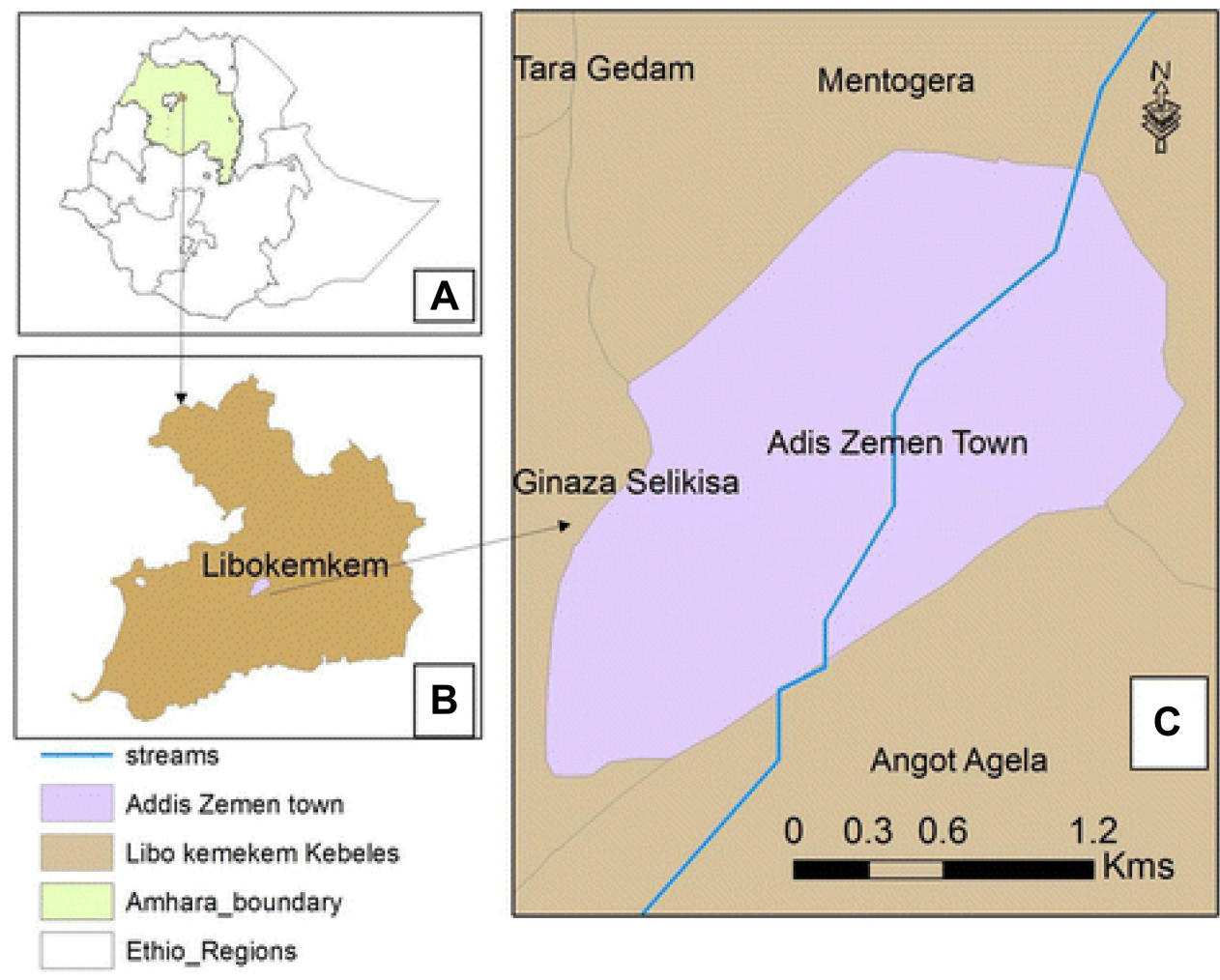

Figure I Map of the study area (Libokemkem district) (source: Kindu et al, 20I8).

\section{Study Design}

An institutional-based retrospective study was conducted to determine five years trend of malaria (2015/16 to 2019/ 20) in Libokemkem district, North West Ethiopia.

\section{Data Collection}

Retrospective data from $2015 / 16$ to $2019 / 20$ on the trend of malaria prevalence were thoroughly collected from the malaria registration book in Addis Zemen health center by the trained data collectors. The sociodemographic information (age, sex and residency), date of blood film examination, total suspected and confirmed cases, months, years and types of malaria species were gathered. In the health center, microscopic examination of Giemsa-stained blood film performed by qualified laboratory technologist was recorded in a malaria registration book from 2015/16 to 2019/20. After preparation of blood film, at least 100 microscope fields were observed and cross-checked by two microscopist who were blind for the result.

\section{Inclusion and Exclusion Criteria}

All suspected and confirmed malaria cases in the study area over a five-year period were included, and any data lacking sociodemographic characteristics, date, month, and year of blood film performed, as well as data lacking species and stage of the parasite were excluded.

\section{Data Quality Control}

The data collectors were trained and given the data extraction tools prior to data collection. Every day, the primary investigator evaluated a sample of the obtained data for completeness and consistency. Finally, a random sample of the extracted data was chosen and examined for completeness, consistency, and accuracy of the data. Before sending data to data entry, the primary investigator counted and documented the number of verified and suspected cases at the end of each data collection.

\section{Operational Definitions Suspected Cases}

A patient with a fever who wanted a blood test because $\mathrm{He} /$ she is suspected of having malaria.

\section{Confirmed Cases}

Patients who have been diagnosed with malaria through blood film. 


\section{Slide Positivity Rate}

The percentage of slides that tested positive for malaria out of the total number of slides analyzed.

\section{Data Analysis}

A data was entered and analyzed using SPSS statistics version 25 . The trends of malaria transmission in terms of seasons, years, and malaria parasite species were depicted using descriptive statistics. The degree of relationship of five-year malaria prevalence with sociodemographic characteristics, seasons, and years was determined using multivariate logistic regressions. Finally, tables and figures were used to present the examined data. Variables with P-values of less than or equal to 0.05 were considered statistically significant.

\section{Ethical Considerations}

Prior to data collection, Debre Tabor University's College of Health Sciences provided ethical clearance (reference number: CHS/230/2012 in Ethiopian calendar) and this study was conducted in accordance with the Declaration of Helsinki. As this was conducted on anonymised secondary data, patient informed consent was waived. Individual names were deleted from the data acquired from the health center to maintain confidentiality, and only unique identification numbers were used to identify individuals.

\section{Results}

\section{Sociodemographic Characteristics of the Study Participants}

At Addis Zemen Health Center, a total of 15, 552 blood films were examined over the study period (2015/162019/20). Males made up 8688 (58.2\%) of the suspected cases, while females made up the rest. Eight thousand and forty-three people $(52.1 \%)$ came from urban areas, while the rest came from rural areas. During the study period, 2094 (13.6\%), 2569 (16.6\%), and 10,789 (69.5\%) of malaria suspected patients were under the age of five, 514 years, and greater than 14 years, respectively. The youngest participant was three months old, and the oldest was 80 years old.

\section{Annual Trends in Total Malaria Cases}

Malaria was found in 1688 of the 15,452 suspected cases using microscopy (10.9\%). On average, 338 cases of malaria were registered annually. The highest proportion of malaria cases was observed in 2019/20 followed by 2015/16, while 2018/19 had the lowest proportion of malaria cases.

The proportion of confirmed malaria cases has steadily decreased from 2015/16 to 2017/18. The proportion of the cases, on the other hand, increased considerably from 2018/19 to 2019/20. In general, the proportion of positive slides declined from $14.0 \%$ in $2015 / 16$ to $5.9 \%$ in $2017 / 18$. It began to fluctuate after that rising to $6.4 \%$ in $2018 / 19$ and then fast to $15.1 \%$ in $2019 / 20$ (Figure 2).

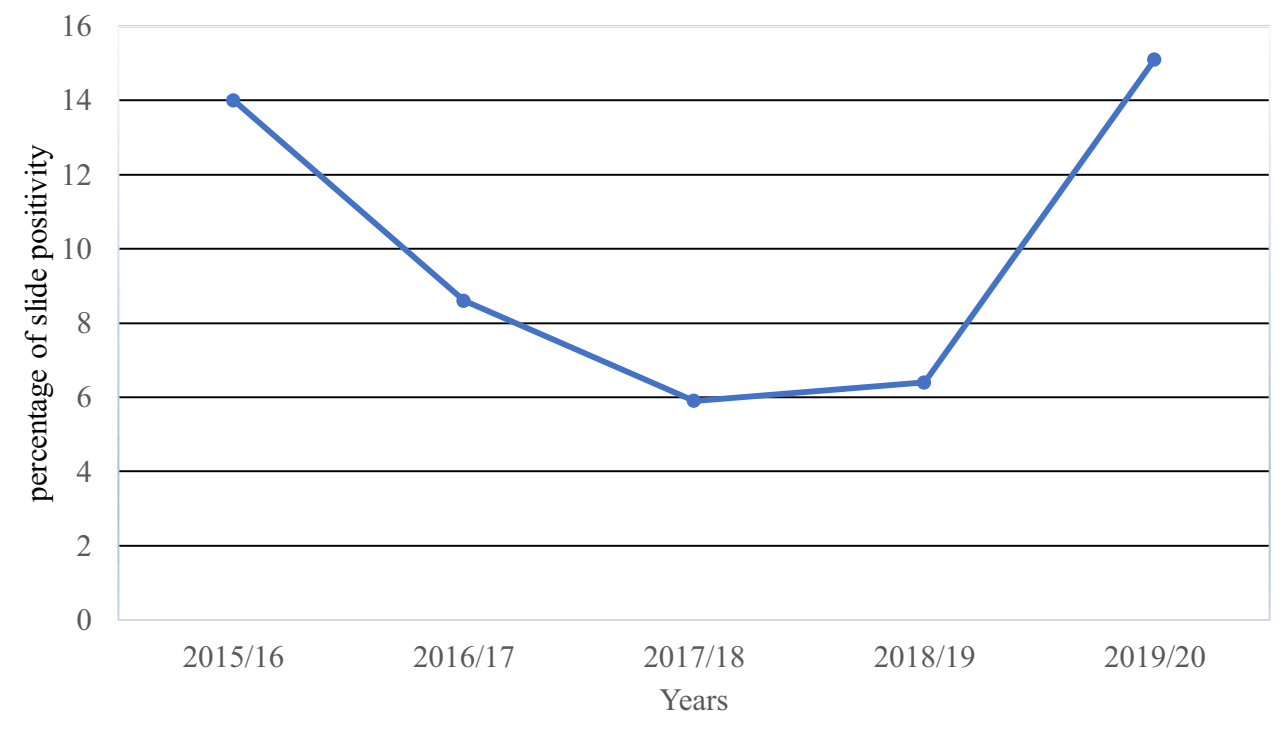

Figure 2 Annual trends of malaria positivity rate in Addis Zemen health center, North west Ethiopia (2015/16-2019/20). 


\section{Plasmodium Species Distribution}

Both $P$. falciparum and $P$. vivax were the only species found in the research area, with $P$. falciparum accounting for 1226 (72.6\%), P. vivax accounting for 441 (26.1\%), and mixed infection accounting for just $21(1.2 \%)$ of the overall prevalence. In this retrospective study, $P$. falciparum was nearly three times more prevalent than $P$. vivax. The prevalence of $P$. falciparum has fluctuated over time, rising from $54.5 \%$ to $80.6 \%$ between $2015 / 16$ and $2017 / 18$. However, at $68.5 \%$ in $2018 / 19$, it exhibited a modest decline. It hit high of $87.8 \%$ in $2019 / 20$.

However, between 2015/16 and 2017/18, the prevalence of $P$. vivax decreased slightly, from $43.7 \%$ to $18.6 \%$. Between 2015/16 and 2019/20, the proportion of mixed infection decreased somewhat, from $1.8 \%$ to $1 \%$ (Figure 3).

\section{Malaria Cases by Sex and Age}

Males were two folds more infected than females, with 1164 and 524 confirmed malaria cases recorded in males and females, respectively, out of 1688 confirmed malaria cases. Males were diagnosed with malaria at a higher rate than females throughout the year (Figure 4).

Patients over the age of 15 years were the most affected age group in this study, with 1229 cases, followed by 5-14-year-olds with 308 cases, while children under the age of five were the least infected by the malaria parasite (Figure 5).

\section{Seasonal Distribution of Malaria}

Malaria cases were reported every month, despite the fact that the disease fluctuates throughout the year. The months with substantial rainfall (June and August), after heavy rainfall (November, October, and September), and May, which is after minor showers of rainfall, had the highest malaria transmission. Malaria cases emerged throughout all seasons, although the highest proportion of malaria cases happened in the summer (June, July, and August) and autumn (September, October, and November), and the lowest case occurred in the winter season (January, February, and March) (Figure 6).

\section{Association of Malaria Trend with Sociodemographic Factors}

Males had a 1.67 higher chance of becoming infected than females over the last five years of malaria prevalence $(\mathrm{AOR}=1.67, \mathrm{CI}=1.49-1.86, \mathrm{P}=0.001)$. Malaria was 1.38 times more prevalent in individuals who lived in rural areas compared to those who lived in urban areas (AOR=1.38 CI=1.25-1.53, p=0.001). Plasmodium parasite infection was 0.59 times less prevalent in children under the age of five than in children over the age of fifteen $(\mathrm{AOR}=0.59, \mathrm{CI}=0.51-0.72, \mathrm{P}=0.001)$. The odds of being infected by Plasmodium spp in 2016/17, 2017/ 18 and 2018/19 were less likely as compared to 2019/ 20 year. The likelihood of having malaria in summer and autumn seasons were 1.60 and 1.51 more as compared to

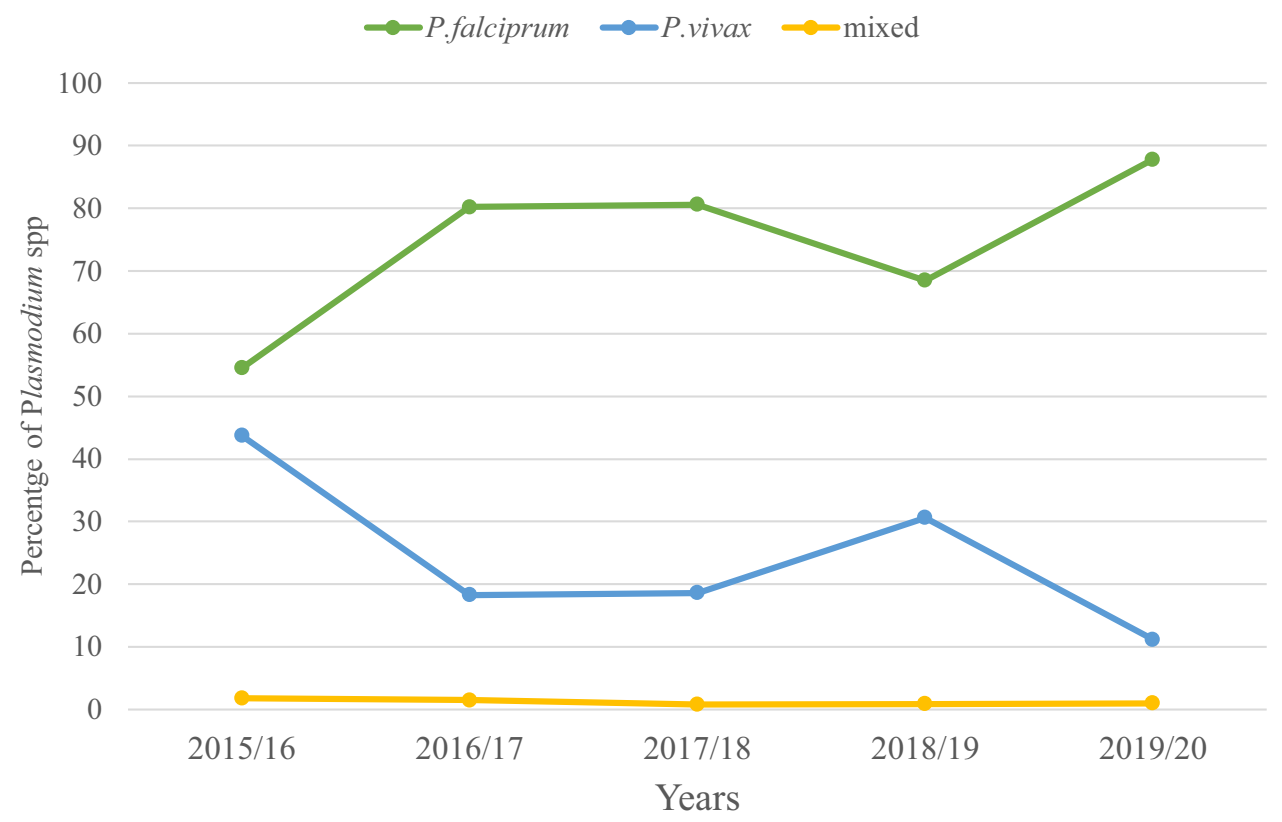

Figure 3 Trends of Plasmodiumspp distribution in Addis Zemen Health Center, North west Ethiopia (2015/16-2019/20). 


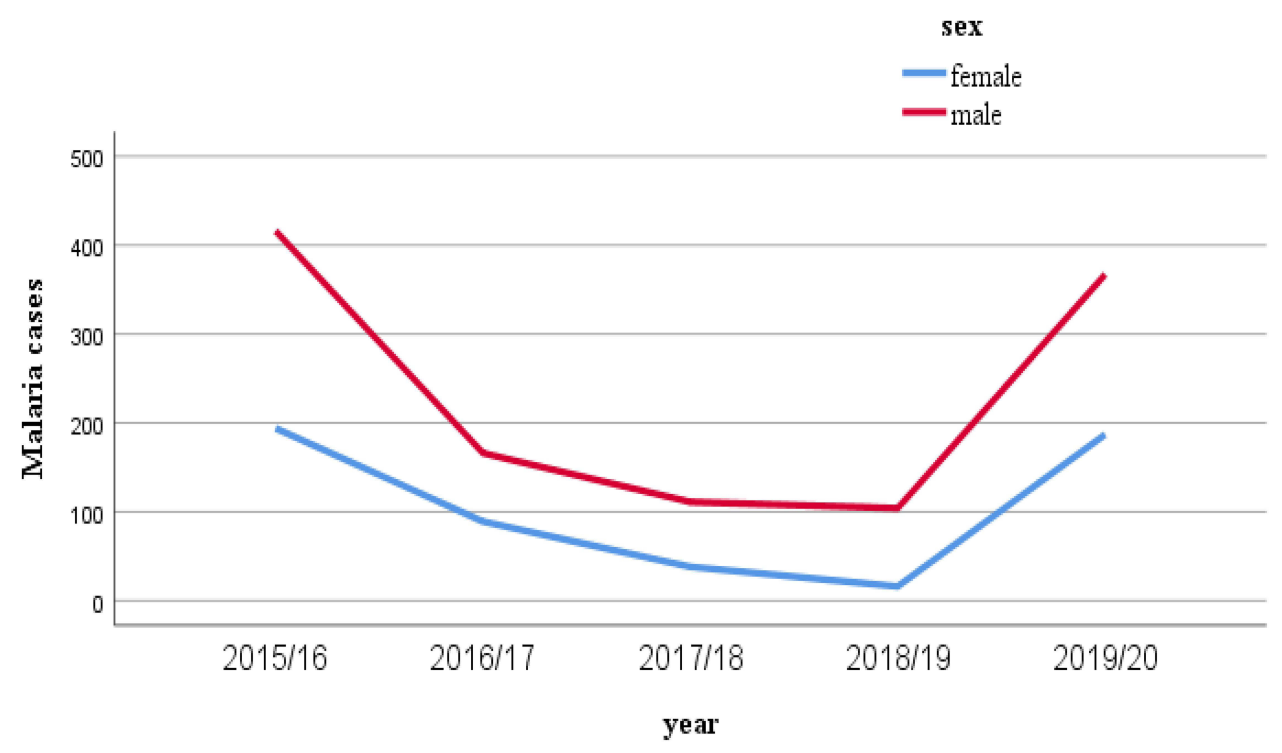

Figure 4 Annual malaria cases by sex distribution in Addis Zemen Health center, North west Ethiopia, (2015/16-2019).

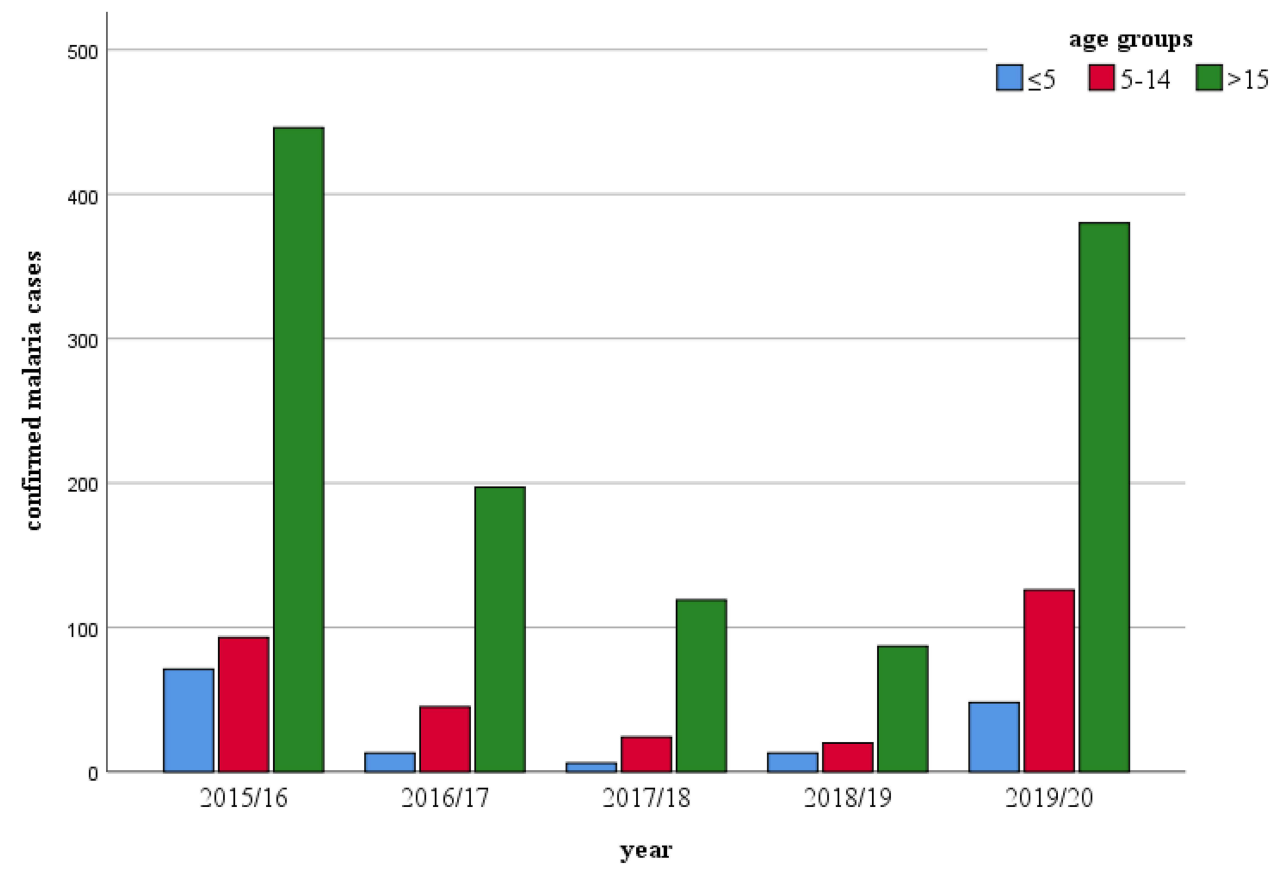

Figure 5 Annual malaria cases by age distribution in Addis Zemen Health center, North West Ethiopia, (2015/16-2019).

winter season $(\mathrm{AOR}=1.60, \mathrm{CI}=1.36-=0.001)$ and $\mathrm{AOR}=$ 1.61, $\mathrm{AOR}=1.51, \mathrm{CI}=1.30-1.76 \mathrm{P}=0.001)$, respectively (Table 1).

\section{Discussion}

Malaria is a major public health concern worldwide, as well as in Ethiopia. Malaria transmission and prevalence vary month to month, season to season, and year to year. As a result, the five-year retrospective analysis undertaken in this study to determine the trend of malaria in Libokemkem district revealed a percentage of 1688/ $15,552(10.9 \%)$. This result was consistent with findings from other investigations. Similar studies in Arsi Negelle Health Center, Kombolcha Health Center, and Dembecha Health Center found that overall prevalence was $11.45 \%$, $7.52 \%$, and $16.34 \%$, respectively. ${ }^{16-18}$ However, this finding was lower than malaria prevalence recorded in Guba district, Boricha District, Southern Ethiopia, and East 


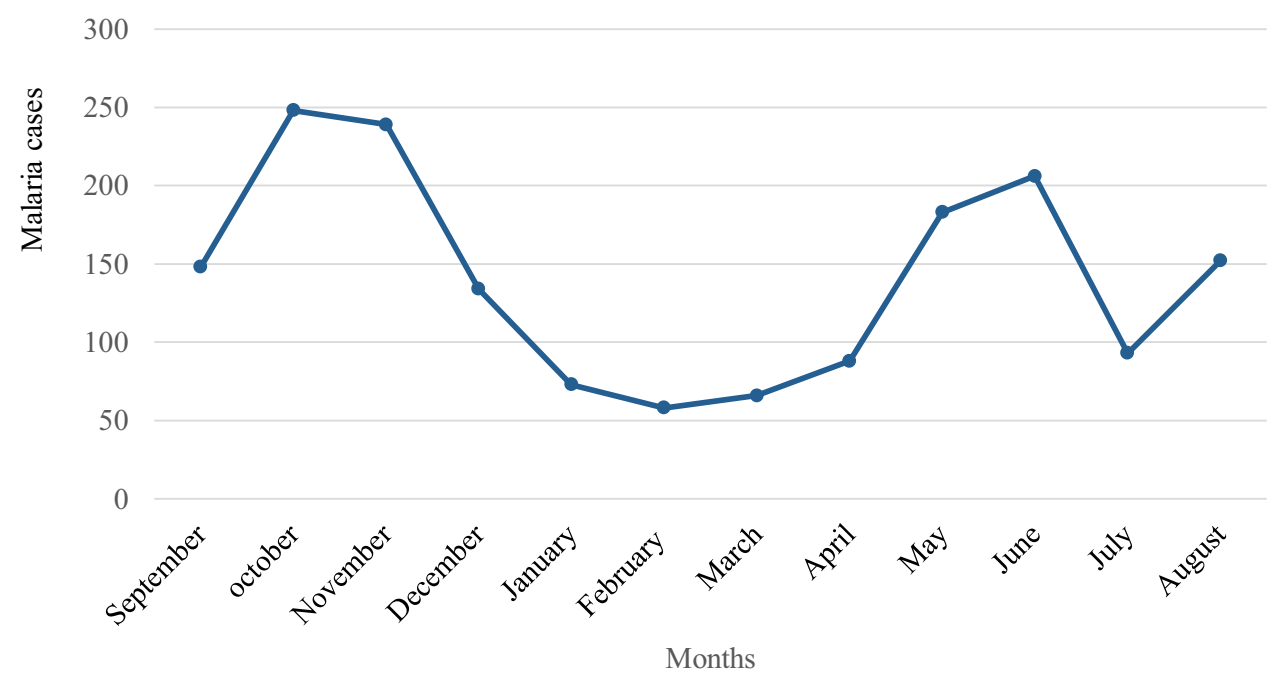

Figure 6 Trend of malaria cases by months in Addis Zemen Health center, North west Ethiopia, (2015/16-2019/20).

Wollega Zone. ${ }^{19-21}$ On the other hand, the research conducted in Felege hiwot referral Hospital and Woreta Health Center, had a lower malaria positivity rate. ${ }^{22,23}$

The discrepancy in malaria positivity rates between this study and others could be attributed to climatic and altitude differences, differences in competency of the microscopists, malaria vector pesticide, and parasite medication resistance. ${ }^{12,13,24}$ The frequency of malaria cases peaked at $14.0 \%$, in $2015 / 16$, but dropped to $6.4 \%$ in 2018 / 19. However, in $2019 / 2020$, it increased to $15.1 \%$. This finding is in line with a research conducted in the Dembia districts. $^{25}$ But, it differs significantly from another researches conducted in Ethiopia. ${ }^{17,19,20,26}$ According to information obtained from the Libokemkem district health office, the cause for the increase in malaria cases in 2019/ 20 is that there have been no intervention efforts such as

Table I Bivariate and Multivariate Logistic Regression Analysis of Factor Associated with Malaria in Addis Zemen Health Center, North West Ethiopia (2015/16-20/9/20)

\begin{tabular}{|c|c|c|c|c|c|c|c|}
\hline \multicolumn{2}{|c|}{ Sociodemographic Characteristics } & \multicolumn{3}{|c|}{ Malaria Status } & \multirow[t]{3}{*}{ COR (Cl) } & \multirow[t]{3}{*}{ AOR (Cl) } & \multirow[t]{3}{*}{ p-value } \\
\hline \multirow[t]{4}{*}{ Sex } & & Positive & Negative & Total & & & \\
\hline & & N (\%) & $\mathrm{N}(\%)$ & $\mathrm{N}(\%)$ & & & \\
\hline & Male & $1164(12.9)$ & $7824(87.1)$ & 8988 (58.2) & $1.69(1.51-1.88)$ & $1.67(1.49-1.86)$ & 0.001 \\
\hline & Female & $524(8.1)$ & $5940(90.8)$ & $6464(41.8)$ & 1 & & \\
\hline \multirow[t]{3}{*}{ Age } & $\leq 5$ & I5I (7.2) & $1943(92.8)$ & $2094(13.5)$ & $0.6 \mathrm{I}(0.5 \mathrm{I}-0.72)$ & $0.59(0.50-0.7 \mathrm{I})$ & 0.001 \\
\hline & $5-14$ & $308(11.9)$ & $226 I(88.1)$ & $2569(16.7)$ & $1.06(0.93-1.21)$ & $1.14(0.99-1.30)$ & 0.068 \\
\hline & $>15$ & $1229(11.3)$ & $9560(88.7)$ & $10,789(69.8)$ & 1 & & \\
\hline \multirow[t]{2}{*}{ Residence } & Urban & $738(9.2)$ & $7305(90.8)$ & $8043(52.1)$ & 1 & & \\
\hline & Rural & $950(12.8)$ & $6459(87.2)$ & $7409(47.9)$ & $1.46(1.32-161)$ & $1.38(1.25-1.53)$ & 0.001 \\
\hline \multirow[t]{5}{*}{ Year } & $2015 / 16$ & $610(14.0)$ & 3761 (86.0) & 4371 (28.3) & $0.98(0.81-1.04)$ & $0.91(0.81-1.04)$ & 0.162 \\
\hline & $2016 / 17$ & $255(8.6)$ & $27 \mid 4$ (9I.4) & $2969(19.2)$ & $0.53(0.45-0.62)$ & $0.54(0.46-0.64)$ & 0.001 \\
\hline & $2017 / 18$ & $149(5.9)$ & $2398(94.1)$ & $2547(16.4)$ & $0.35(0.29-0.43)$ & $0.34(0.28-0.42)$ & 0.001 \\
\hline & $2018 / 19$ & $120(6.4)$ & 1756 (93.6) & $1876(12.1)$ & $0.388(0.430 .48)$ & $0.40(0.33-0.50)$ & 0.001 \\
\hline & $2019 / 20$ & $554(15.1)$ & $3135(84.9)$ & $3689(23.9)$ & 1 & & \\
\hline \multirow[t]{4}{*}{ Season } & Winter & $265(8.3)$ & 2920 (91.7) & $3185(20.6)$ & 1 & & \\
\hline & Spring & $337(8.9)$ & 3444 (9I.I) & $3781(24.5)$ & $1.08(0.91-1.28)$ & $1.12(0.94-1.33)$ & 0.199 \\
\hline & Summer & $45 I(12.8)$ & 3063 (97.2) & $3514(22.7)$ & $1.62(1.38-1.90)$ & $1.60(1.36-1.88)$ & 0.001 \\
\hline & Autumn & $635(12.5)$ & 4337 (87.5) & $4972(32.2)$ & $1.61(1.38-1.88)$ & $1.51(1.30-1.76$ & 0.001 \\
\hline
\end{tabular}


availability of ITN for the past three years in the district. Furthermore, there may be persistence of the parasite due to drug resistance in the area.

P. falciparum and P. vivax accounted for $72.6 \%$ and $26.1 \%$ of the overall reported cases, respectively, with the rest being mixed illness. This data is consistent with the distribution of Plasmodium spp. described in different locations of Ethiopia. ${ }^{16,17,20}$ The most common parasite found was $P$. falciparum, which fluctuated during the study period. The fact that $P$. falciparum multiplies quickly, infects all ages of red blood cells, and is resistant to antimalarial medicines may explain its dominance over P.vivax. ${ }^{1,12}$

Months and seasonality play a role in malaria transmission. Malaria cases were found in every month of the year in this study, although the highest number of cases was found in October, November, June, August, and September. In comparison to dry season (winter), both summer (June, July, and August) and autumn (September, October, and November) had the highest transmissions rate. This finding was consistent with findings from other investigations conducted Ethiopian. ${ }^{18,19,22,26}$ The finding also corresponds to the monthly malaria transmission pattern reported by Ethiopia's federal ministry of health. ${ }^{27}$ This is because high rainfall in the summer creates a perfect breeding habitat for female Anopheles mosquitos, causing malaria vector density to peak in most parts of the country from summer to autumn.

Males were 1.69 times more likely than females to contract malaria in this retrospective analysis. Other studies done in Ethiopia ${ }^{18,20,26,28}$ revealed greater malaria cases among males in a similar fashion. Males are more likely to contract malaria due to their involvement in outdoor activities such as agricultural and pastoral activities, as well as remaining outside during exophagic mosquito biting hours.

In terms of age groupings, the burden of malaria morbidity was found in the age group of $>15$ years, while children under the age of five were 0.59 less likely to be infected by Plasmodium spp than adults. Other studies have previously shown similar findings. ${ }^{18-20,22}$

The explanation for this could be because they engage in outdoor activities since they are responsible for caring for the family, and children under the age of five are less likely to be exposed to mosquitoes due to parental/guardian awareness of malaria prevention and control.

In comparison to urban people, rural dwellers were 1.38 times more likely to contract malaria. Inadequate understanding about malaria among rural communities, insufficient ITN coverage, the availability of an ecological niche conducive to mosquito reproduction like stagnant water, and participation in outdoor activities could all be contributory causes.

\section{Limitation}

We were unable to get malaria data from the health center for more than five years in order to conduct a retrospective analysis of more than five years.

\section{Conclusion}

Malaria control program improvement was observed from $2016 / 17$ to $2018 / 19$, as malaria morbidity decreased over this time period. However, there was high morbidity case in 2019/20. Therefore, raising awareness about malaria control and prevention, as well as malaria intervention programs such as providing ITN to all communities in the district and training laboratory personnel on proper parasite identification, should be strengthened in the district in order to meet the Ethiopian Ministry of Health's malaria elimination goal.

\section{Acknowledgments}

We'd like to thank the coordination office of the Addis Zemen health center for letting us to collect the data. We also want to express our gratitude to the data collectors.

\section{Disclosure}

The authors declare no competing of interest.

\section{References}

1. Gillespie S, Pearson RD. Principles and Practice of Clinical Parasitology. John Wiley \& Sons; 2003.

2. Ayele DG, Zewotir TT, Mwambi HG. Prevalence and risk factors of malaria in Ethiopia. Malar J. 2012;11(1):195. doi:10.1186/1475-2875-11-195

3. Satoskar A, Simon G, Hotez P, Tsuji M. Medical Parasitology. Texas: Landes Bioscience; 2009.

4. Erkins DJ, Were T, Davenport GC, Kempaiah P, Hittner JB, Ong'echa JM. Severe malarial anemia: innate immunity and pathogenesis. Int J Biol Sci. 2011;7(9):1427. doi:10.7150/ijbs.7.1427

5. World Malaria Report 2020. 20 Years of Global Progress and Challenges. Geneva: World Health Organization; 2020. Licence: CC BY-NC-SA 3.0 IGO.

6. egegne Y, Asmelash D, Ambachew S, Eshetie S, Addisu A, Jejaw Zeleke A. The prevalence of malaria among pregnant women in Ethiopia: a systematic review and meta-analysis. Journal of parasitology research. 2019; doi:10.1155/2019/8396091

7. World Health Organization (WHO). World malaria report 2015. Geneva: World Health Organization; 2015. Available from: http:// www.who.int/malaria/visual-refresh/en/. Accessed August 21, 2021.

8. Adugna A. Malaria in Ethiopia. Available from: http://www.ethiode mographyandhealth.org/MedVectoredDiseasesMalaria.pdf. Accessed December 4, 2013.

9. President's malaria initiative. Malaria Operational Plan FY 2014. Ethiopia: President's Malaria Initiative; 2014. 
10. Ethiopian Federal ministry of Health. Ethiopia Malaria Elimination Strategic Plan: 2021-2025. Available from: http://www.moh.gov.et/ ejcc/am/ETHIOPIA_MALARIA_ELIMINATION_STRATEGIC_ PLAN: 2021-2025. Accessed December 04, 2021.

11. Ethiopian Public Health Institute. Ethiopia national malaria indicator survey 2015. Available from: https:/www.ephi.gov.et/images/pic tures/download2009/MIS-2015-Final-Report-December-_2016.pdf. Accessed September 3, 2021.

12. Bloland PB; World Health Organization. Drug Resistance in Malaria. World Health Organization; 2001.

13. Alout H, Roche B, Dabiré RK, Cohuet A. Consequences of insecticide resistance on malaria transmission. PLoS Pathog. 2017;13(9): e1006499. doi:10.1371/journal.ppat.1006499

14. CSA (Central Statistics Authority). Summary and Statistical Report of the 2007 Population and Housing Census. Addis Ababa, Ethiopia: Population Size by Age and Sex; 2008.

15. Kindu M, Aklilu E, Balkew M, Gebremichael T. A study on the species composition and ecology of anopheline in Addis Zemen, South Gondar, Ethiopia. Parasit Vectors. 2018;11(1):215 doi:10.1186/s13071-018-2701-3

16. Hailemariam M, Gebre S. Trend analysis of malaria prevalence in Arsi Negelle health center, Southern Ethiopia. J Infect Dis Immun. 2015;7(1):1-6. doi:10.5897/JIDI2014.0147

17. Gebretsadik D, Feleke DG, Fiseha M. Eight-year trend analysis of malaria prevalence in Kombolcha, South Wollo, north-central Ethiopia: a Retrospective Study. Parasit Vectors. 2018;11(1):1-6. doi:10.1186/s13071-018-2654-6

18. Haile D, Ferede A, Kassie B, Abebaw A, Million Y. Five-year trend analysis of malaria prevalence in Dembecha Health Center, West Gojjam Zone, Northwest Ethiopia: a Retrospective Study. J Parasitol Res. 2020;2020:1-7. doi:10.1155/2020/8828670

19. Alkadir S, Gelana T, Gebresilassie A. A five-year trend analysis of malaria prevalence in Guba district, Benishangul-Gumuz regional state, western Ethiopia: a Retrospective Study. Trop Dis Travel Med. 2020;6(1):1-7. doi:10.1186/s40794-020-00112-4
20. Dabaro D, Birhanu Z, Yewhalaw D. Analysis of trends of malaria from 2010 to 2017 in Boricha District, Southern Ethiopia. Malar J. 2020;19(1):1. doi:10.1186/s12936-020-03169-w

21. Babure ZK, Ahmed YM, Likasa ST, Jiru FA, Weldemarium TD, Fite MB. Trend analysis of malaria prevalence in East Wollega Zone, Oromia Regional State, Western Ethiopia, 2020: a Retrospective Study. J Womens Health Care. 2021;10(515):21 67-2420.

22. Yimer M, Hailu T, Mulu W, Abera B, Ayalew W. A 5 year trend analysis of malaria prevalence with in the catchment areas of Felegehiwot referral Hospital, Bahir Dar city, northwest-Ethiopia: a Retrospective Study. BMC Res Notes. 2017;10(1):1-4. doi:10.11 86/s13104-017-2560-6

23. Derbie A, Alemu M. Five years malaria trend analysis in Woreta health center, Northwest Ethiopia. Ethiop J Health Sci. 2017;27 (5):465-472. doi:10.4314/ejhs.v27i5.4

24. Paaijmans KP, Blanford S, Bell AS, Blanford JI, Read AF, Thomas MB. Influence of climate on malaria transmission depends on daily temperature variation. Proc Natl Acad Sci U S A. 2010;107 (34):15135-15139. doi:10.1073/pnas.1006422107

25. Addisu A, Tegegne Y, Mihiret Y, Setegn A, Zeleke AJ. A 7-year trend of malaria at primary health facilities in Northwest Ethiopia. J Parasitol Res. 2020;2020:1-5. doi:10.1155/2020/4204987

26. Molla E, Behaksra SW, Tadesse FG, Dugassa S, Gadisa E, Mamo H. Past eight-year malaria data in Gedeo zone, southern Ethiopia: trend, reporting-quality, spatiotemporal distribution, and association with socio-demographic and meteorological variables. BMC Infect Dis. 2021;21(1):1-5. doi:10.1186/s12879-021-05783-8

27. Federal Democratic Republic of Ethiopia Ministry of Health. National Malaria Elimination Strategic Plan: 2021-2025. Addis Ababa:FMOH; 2020:1-76.

28. Beyene B, Gelana T, Gebresilassie A. Five years trend analysis of malaria prevalence in JardgaJarte District, Western Ethiopia. Sinet Ethiop J Sci. 2018;41(2):61-69.
Infection and Drug Resistance

\section{Publish your work in this journal}

Infection and Drug Resistance is an international, peer-reviewed openaccess journal that focuses on the optimal treatment of infection (bacterial, fungal and viral) and the development and institution of preventive strategies to minimize the development and spread of resistance. The journal is specifically concerned with the epidemiology of

\section{Dovepress}

antibiotic resistance and the mechanisms of resistance development and diffusion in both hospitals and the community. The manuscript management system is completely online and includes a very quick and fair peerreview system, which is all easy to use. Visit http://www.dovepress.com/ testimonials.php to read real quotes from published authors. 